\title{
Impact of cardiac surgery on the autonomic cardiovascular function
}

\author{
Sebastian Zaunseder ${ }^{{ }^{*}}$, Maik Riedl ${ }^{2}$, Jürgen Kurths ${ }^{3}$, Hagen Malberg ${ }^{1}$, Robert Bauernschmitt ${ }^{4}$ and \\ Niels Wessel ${ }^{2}$
}

\author{
*Correspondence: sebastian. \\ zaunseder@tu-dresden.de \\ ${ }^{1}$ Institute of Biomedical \\ Engineering, TU Dresden, \\ Helmholtzstraße 18, Dresden 01069, \\ Germany \\ Full list of author information is \\ available at the end of the article
}

\begin{abstract}
Background: The study compares the impact of cardiac surgical interventions on the autonomic function by assessing the pre-operative status and early post-operative recovery of subjects undergoing isolated mitral valve replacement (MVR), isolated aortic valve replacement (AVR), and transcatheter aortic valve implantation (TAVI). We analyze heart rate variability (HRV), baroreflex sensitivity (BRS), and cardiovascular coupling in a longitudinal, i.e., the temporal evolution of autonomic function within each group before, 1 day after, and 7 days after surgery, and a transversal, i.e., between groups of patients at identical time instants, setting.

Results: A total of 243 records from 124 patients (38 MVR, 57 AVR, 29 TAVI) was analyzed. There were no major differences in HRV, BRS, and coupling between the groups in the pre-operative values. Longitudinal analysis proves a depressed autonomic function for MVR and AVR patients after surgery (in MVR patients, $p<0.001$ for most parameters related to HRV and BRS), but not for TAVI patients. TAVI patients showed no differences before and after surgery. Transversal analysis reveals the strongest impairments throughout HRV and BRS parameters for MVR patients. In the case of AVR, the autonomic regulation was also depressed, though not to the extent as seen in MVR patients. Cardiovascular coupling by means of symbolic coupling traces (SCT) was shown to be clearly reduced the day after surgery in MVR and AVR patients. In TAVI patients, there was no reduction but already the day after surgery developed additional couplings.
\end{abstract}

Conclusions: Our results prove a characteristic behavior of the autonomic function in relation to the gravity of the surgical procedure. As variables related to the process of the surgical interventions were kept similar between patient groups, direct surgical trauma is assumed to be responsible for the heavy decrease of autonomic function in the case of MVR. TAVI, in contrast, proves to be very suited in terms of maintaining the autonomic function in comparison to AVR. Further studies incorporating larger populations should confirm our findings and relate the autonomic state to malignant events after surgical interventions to build the fundament of a strengthened inclusion of cardiovascular variability and coupling analysis in the pre-, peri-, and post-operative care.

Keywords: Autonomic function; Heart rate variability; Baroreflex sensitivity; Cardiovascular coupling; Aortic valve replacement; Mitral valve replacement; Transcatheter aortic valve implantation

\section{Springer}

(c) 2014 Zaunseder et al.; licensee Springer. This is an Open Access article distributed under the terms of the Creative Commons Attribution License (http://creativecommons.org/licenses/by/2.0), which permits unrestricted use, distribution, and reproduction in any medium, provided the original work is properly cited. 


\section{Background}

The autonomic function has been shown to be a strong predictor of cardiac mortality after myocardial infarction [1]. An increased sympathetic activity and/or a reduced vagal activity, quantified by means of baroreflex sensitivity (BRS) and heart rate variability (HRV), respectively, were found to be independent prognostic markers [2]. Even for patients without a history of heart failure, an impaired vagal tone was shown to be predictive of fatal cardiac events [3].

Against this background, even in the context of surgery, the autonomic function has attracted interest. In pre-, peri-, and post-operative settings, a detailed analysis of the cardiovascular variability may contribute to an improved care by identifying persons at risk, adjust required interventions to individual needs, and assess short- and long-term trends to guide therapeutic actions after surgery. However, although the basic knowledge about the autonomic cardiovascular function has been continuously growing in the last decades, alterations in the cardiovascular variability related to cardiac surgery remain to be characterized in the future. Such a characterization is difficult as different influence factors most importantly anesthesia, the use of the heart-lung machine, and direct surgical trauma - are assumed to possibly affect the cardiovascular variability.

Most previous work which is related to the impact of cardiac surgery on the autonomic function was directed at coronary artery bypass surgery. Laitio et al. [4] proved a drop in HRV $24 \mathrm{~h}$ after surgery. Bauernschmitt et al. [5] confirmed this drop for various measures of autonomic cardiovascular function including parameters of heart rate variability, BRS, and blood pressure variability (BPV). Soares et al. [6] expanded the observational interval and could show that after an early drop there occurs a recovery of autonomic function after 30 days. Johannson et al. [7] reported a partial recovery after 5 months while 5 weeks after surgery the autonomic function kept on being impaired. A similar time frame was described by Demirel et al. [8] who reported a recovery in HRV parameters to occur 3 months after surgery. Less work has spent on the impairment after cardiac valve surgery so far. In general, an impairment of autonomic function similar to the one after coronary artery bypass surgery can be expected. However, the degree of impairment can be assumed to vary with the type of intervention, i.e., which valve is concerned, and chosen operational procedure. In this regard, Lakusic et al. [9] showed that the impairment in autonomic function 3.8 months after surgery was much more pronounced for mitral valve implantation compared to aortic valve implantation.

Our own work now seeks to deepen the understanding of autonomic impairment immediately after cardiac valve surgery. The presented contribution compares the impact of different surgical procedures on the autonomic cardiovascular regulation by assessing the preoperative status and early post-operative recovery of subjects undergoing isolated mitral valve replacement (MVR), isolated aortic valve replacement (AVR), and transcatheter aortic valve implantation (TAVI) whose feasibility and benefits, particularly considering high-risk patients, have been demonstrated [10,11]. By comparing surgical interventions of differing gravity, we try to confirm the hypothesis of a likewise impairment of the autonomic function by analyzing cardiovascular variability and coupling.

We previously analyzed the patients undergoing the depicted interventions by means of their HRV, BPV, and BRS in pairwise settings, i.e., AVR vs MVR and AVR vs TAVI [12,13]. The present contribution pooled together and widened the mentioned studies in terms of implicated patients, applied methods, and finally drew conclusions. That is to say, we 
review major findings related to HRV and BRS in the face of a more complete patient collective and complement such considerations by the analysis of cardiovascular coupling between the heart rate (HR) and blood pressure (BP) by means of symbolic coupling traces (SCT) [14] which are applied for the first time in the presented setting.

\section{Methods}

\section{Surgical procedures}

For MVR and AVR, peri-operative medication as well as anesthesia was standardized. Induction was performed with sufentanil and midazolam. For maintaining narcosis, a continuous infusion of propofol was given. Muscle relaxation was achieved by pancuronium. Operations were carried out with cardiopulmonary bypass in mild hypothermia $\left(32^{\circ} \mathrm{C}\right.$ to $\left.34^{\circ} \mathrm{C}\right)$ and pulsatile perfusion mode; cold crystalloid cardioplegia was used for cardiac arrest after cross-clamping the aorta. Surgical access to the AV was achieved by horizontal transection of the anterior aspect of the ascending aorta. Access to the MV was performed by opening the left atrium close to the interatrial groove. After declamping, most of the patients needed one countershock to terminate ventricular fibrillation.

TAVI was performed with the patients under general anesthesia. Pharmacologic treatment was analogous to the surgical interventions except for muscle relaxation, which was not applied in transcatheter patients. TAVI was conducted either by transfemoral access or through the left ventricular apex by a surgical team in a hybrid suite [15]. After the procedure, the patients were transferred to the intensive care unit and usually extubated within 2 to $4 \mathrm{~h}$. Post-operative medical and pharmacologic care was again adapted to the MVR and AVR patients as far as possible.

\section{Recording protocol and subjects}

Thirty-minute recordings in supine position were acquired in three sessions: before (preOP), 1 day after ( $1 \mathrm{~d}$ postOP), and 7 days after ( $7 \mathrm{~d}$ postOP) surgery. From each patient and session, we analyzed signal segments of $20 \mathrm{~min}$ following the first $10 \mathrm{~min}$ of equilibration and familiarization to the recording setup. Measurements comprised a single-channel electrocardiogram (ECG), respiration (i.e., breathing excursion), and noninvasive continuous blood pressure. The latter was acquired either from the radial artery (Colin Medical Instruments, San Antonio, TX, USA) or recorded by using a finger cuff (Task Force Monitor, CNSystems, Graz, Austria). Patients with concomitant coronary heart disease and those who did not show stable sinus rhythm were excluded. Table 1 gives a summary on available patients and surgical parameters.

The study was approved by the local ethics committee of the Technical University of Munich. Informed consent was obtained from all subjects.

The times between consecutive R-peaks were extracted from the ECG. From the continuous BP, beat-to-beat systolic blood pressure (SBP) was extracted. Premature beats, artifacts, and noise were excluded using an adaptive filter considering the instantaneous variability of both beat-to-beat intervals and beat-wise SBP, respectively [16].

\section{Analysis of the autonomic function}

Univariate analysis of HRV

HRV describes the variability of the instantaneous HR, i.e., the variability between intervals of consecutive normal heart beats (beat-to-beat intervals, BBI). Analysis of HRV 
Table 1 Patient characteristics

\begin{tabular}{llcccc}
\hline & & MVR & AVR & TAVI & p value \\
\hline Patients (preOP/1d postOP/7d postOP) & $31 / 22 / 19$ & $50 / 37 / 30$ & $24^{\text {a }} / 16 / 14$ & - \\
Gender (m/f) & preOP & $22 / 9$ & $40 / 10$ & $7 / 17$ & $<0.001$ \\
& 1d postOP & $17 / 5$ & $30 / 7$ & $4 / 12$ & $<0.001$ \\
& 7d postOP & $18 / 1$ & $24 / 6$ & $4 / 10$ & $<0.001$ \\
Age & preOP & $60 \pm 13$ & $63 \pm 13$ & $80 \pm 7$ & $<0.001$ \\
(mean \pm sd in years) & 1d postOP & $58 \pm 12$ & $62 \pm 12$ & $81 \pm 8$ & $<0.001$ \\
& 7d postOP & $60 \pm 11$ & $60 \pm 12$ & $79 \pm 9$ & $<0.001$ \\
Hypertension & preOP & 15 & 25 & 14 & n.s. \\
& 1d postOP & 7 & 18 & 9 & n.s. \\
Pulmonary disease & 7d postOP & 8 & 17 & 9 & n.s. \\
& preOP & 2 & 3 & 4 & n.s. \\
Thyroid dysfunction & 1d postOP & 1 & 1 & 1 & n.s. \\
& 7d postOP & 1 & 0 & 2 & n.s. \\
& preOP & 3 & 1 & 1 & n.s. \\
Time of surgery & 1d postOP & 2 & 1 & 1 & n.s. \\
(mean \pm sd in min) & 7d postOP & 0 & 1 & 0 & n.s. \\
& preOP & $224 \pm 63$ & $224 \pm 83$ & $102 \pm 35$ & $<0.001$ \\
& 1d postOP & $207 \pm 56$ & $215 \pm 66$ & $98 \pm 28$ & $<0.001$ \\
7d postOP & $200 \pm 49$ & $217 \pm 70$ & $95 \pm 31$ & $<0.001$ \\
\hline
\end{tabular}

Patient characteristics after removal of patients/records which met an exclusion criterion. A total of 243 records from 124 patients (38 MVR, 57 AVR, 29 TAVI) was analyzed. We are reporting on 38 patients with MVR, 17 of whom appeared in [12]; on 57 patients with AVR, 26 and 34 of whom appeared in [12] and [13], respectively; and on 29 patients with TAVI, 24 of whom appeared in [13]. $p$ values for categories 'age' and 'time of surgery' resulted from the Kruskal-Wallis test; for the remaining categories, the cross-table $\chi^{2}$ test of independence was applied. Note that the characteristics within the groups change accordingly the patients which could be used at a given time instant. ${ }^{a}$ In 15 patients, transfemoral access was performed and in 14 patients, through the left ventricular apex (overall 29 patients). At time instants preOP, $1 \mathrm{~d}$ postOP, and $7 \mathrm{~d}$ postOP, 12,8 , and 8 transfemoral patients and 12,8 , and 6 patients were included into the analysis. ${ }^{\text {bThe information was }}$ available for 29/22/19 (MVR), 42/33/28 (AVR), and 18/14/14 (TAVI) patients. sd, standard deviation; n.s., not significant.

can be done using various parameters from different domains. A fundamental grading of approaches to analyze HRV was introduced by the Task Force in 1996 [17]. In [17] time domain, frequency domain, and non-linear measures are distinguished.

Our analysis incorporated parameters from the three aforementioned domains. HRV parameters were derived from the original time series, either after applying a standard Fourier transform or after applying a symbolic transform [18]. Regarding the latter, two variants were used. On the one hand, a transform was used which yields a binary symbolic representation $s 1_{x}(n)$. From an input denoted as $x(n)$, the binary symbol sequence $s 1_{x}(n)$ was derived by applying the rule

$$
s 1_{x}(n)=\left\{\begin{array}{l}
1 \text { if }|x(n)-x(n-1)| \geq 10 \mathrm{~ms} \\
0 \text { if }|x(n)-x(n-1)|<10 \mathrm{~ms}
\end{array}\right.
$$

On the other hand, we applied a transform which yields a multivariate representation. The multivariate symbol sequence $s 2_{x}(n)$ resulted from

$$
s 2_{x}(n)= \begin{cases}0 \text { if } \mu & <x(n) \leq(1+a) \cdot \mu \\ 1 \text { if }(1+a) \cdot \mu & <x(n)<\quad \infty \\ 2 \text { if }(1-a) \cdot \mu & <x(n)<\quad \mu \\ 3 \text { if } 0 & <x(n) \leq(1-a) \cdot \mu\end{cases}
$$

where $\mu$ denotes the mean value of $x(n)$, i.e., the mean BBI, and $a$ equals 0.05 [16]. From $s 1_{x}$ and $s 2_{x}$, words of six and three symbols, respectively, were composed. From the 
resulting word sequences, two parameters - POLVAR10, the probability of low variability, and FORBWORD, the number of forbidden words - were derived. POLVAR10 was calculated by relating the number of words which show no variability, i.e., 000000 , to all words. FORBWORD counts all three-symbol words which occur never or seldomly, i.e., one occurrence or no occurrence.

Table 2 summarizes all parameters which have been analyzed to characterize the HRV along with their meanings. Note that shannon is derived directly from the histogram of the time series data using the expression

$$
\text { shannon }=-\sum_{i} p_{i} \log p_{i}
$$

where $p_{i}$ are the bins of the BBI histogram. More details regarding the calculation of the used parameters and their informative value can be found in [17] and [16]. Retzlaff et al. $[12,13]$ applied some additional univariate features (selected HRV features and features related to the BPV) which, due to the uniformity of the results obtained by using those features compared to the selected ones, were not separately considered in this contribution.

\section{Bivariate analysis}

Bivariate methods exploit the interdependency of the instantaneous HR and the beatto-beat BP. Although bivariate techniques have been in the focus of researchers since decades, bivariate analysis still shows considerable methodological progress. Nowadays, of particular interest are methods which allow for a proper information retrieval in the face of typical biological phenomena such as non-linearity and instationarity. As bivariate methods, we considered for our analysis BRS and coupling phenomena.

Parameters related to BRS were extracted by using the dual sequence method (DSM). Similar to standard sequence methods (e.g., [19]), DSM exploits slopes in blood pressure and heart rate. DSM assesses bradycardic (an increase in SBP that causes an increase in BBI) and tachycardic (a decrease in SBP that causes a decrease in BBI) fluctuations (see Figures 1 and 2) in a synchronous and in a shifted mode. Thereby, bradycardic fluctuations are attributed to the vagal spontaneous baroreflex whereas tachycardic fluctuations allow investigations on the relationship of vagally and sympathetically mediated fluctuations in BBI [20]. For this contribution, both types of fluctuation were analyzed in a synchronous and in a three-beat shifted mode. The most important parameters which were calculated were the mean slopes of BRS (tachycardic BRS and bradycardic BRS, both in $\mathrm{ms} / \mathrm{mmHg}$ ).

Table 2 Used HRV parameters

\begin{tabular}{|c|c|c|c|c|}
\hline Domain & & Parameter & Description & Unit \\
\hline \multirow{5}{*}{ Time domain } & \multirow{2}{*}{ Linear } & meanNN & Mean value of the beat-to-beat intervals & ms \\
\hline & & sdNN & Standard deviation of the beat-to-beat intervals & $\mathrm{ms}$ \\
\hline & & shannon & Shannon entropy of the beat-to-beat intervals & Unitless \\
\hline & \multirow[t]{3}{*}{ Non-linear } & FORBWORD & Number of forbidden words & Unitless \\
\hline & & POLVAR10 & Probability of low variability $<10 \mathrm{~ms}$ & Unitless \\
\hline & & $P_{\mathrm{LF} / P}$ & Normalized low-frequency power (LF 0.04 to $0.15 \mathrm{~Hz}$ ) & Unitless \\
\hline \multirow{2}{*}{\multicolumn{2}{|c|}{ Frequency domain }} & $P_{\mathrm{HF} / P}$ & Normalized high-frequency power (HF 0.15 to $0.4 \mathrm{~Hz}$ ) & Unitless \\
\hline & & $P_{\mathrm{LF}} / P_{\mathrm{HF}}$ & Ratio of LF power to HF power & Unitless \\
\hline
\end{tabular}

Parameters used to quantify HRV. Details on the calculation can be found in [17] and [16]. 


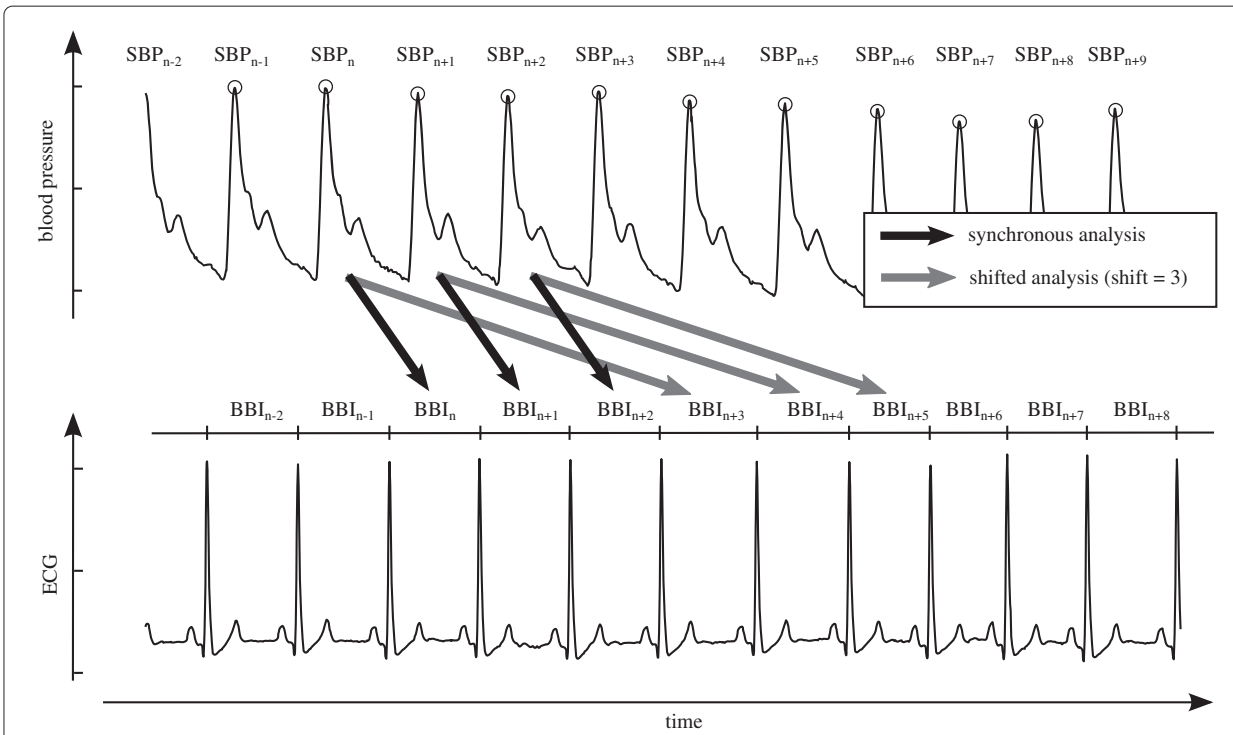

Figure 1 DSM basics. Basic scheme to assess the baroreflex sensitivity by means of DSM. DSM evaluates synchronous and shifted slopes in HR and SBP. The example considers slopes of three consecutive beats in a synchronous and a shifted mode. See [20] for details.

Coupling was assessed by analyzing symbolic coupling traces (SCT). In general, the analysis of cardiovascular couplings targets the interconnection between HR and BP by finding a pattern in both signals which exhibit mutual causality. SCT particularly was developed by Wessel et al. to quantify couplings in symbolic time series by means of their bivariate word distribution [14]. Figure 3 outlines the scheme underlying SCT: from a symbolic representation of two time series $x(n)$ and $y(n)$ derived by

$$
s_{z}(n)=\left\{\begin{array}{l}
1 \text { if } z(n) \leq z(n+\vartheta) \\
0 \text { if } z(n)>z(n+\vartheta)
\end{array}\right.
$$

words of length $l$ are composed. The occurrence of words in the respective word sequence $w_{x}(n)$ and $w_{y}(n)$ builds up a bivariate word distribution. The word distribution shows the probability of a joint occurrence of two words $W_{i}$ and $W_{j}$ in $w_{x}(n)$ and $w_{y}(n)$. By introducing a time lag $\tau$ (with $\tau$ restricted to integer numbers), time shifts within the

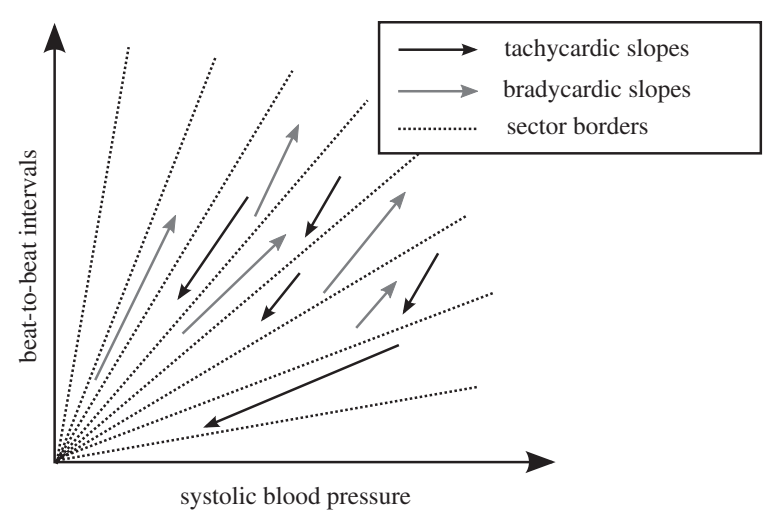

Figure 2 Slopes considered by DSM. Analysis of relevant slopes by using DSM. DSM exploits tachycardic and bradycardic slopes which can be further analyzed in sectors. See [20] for details. 


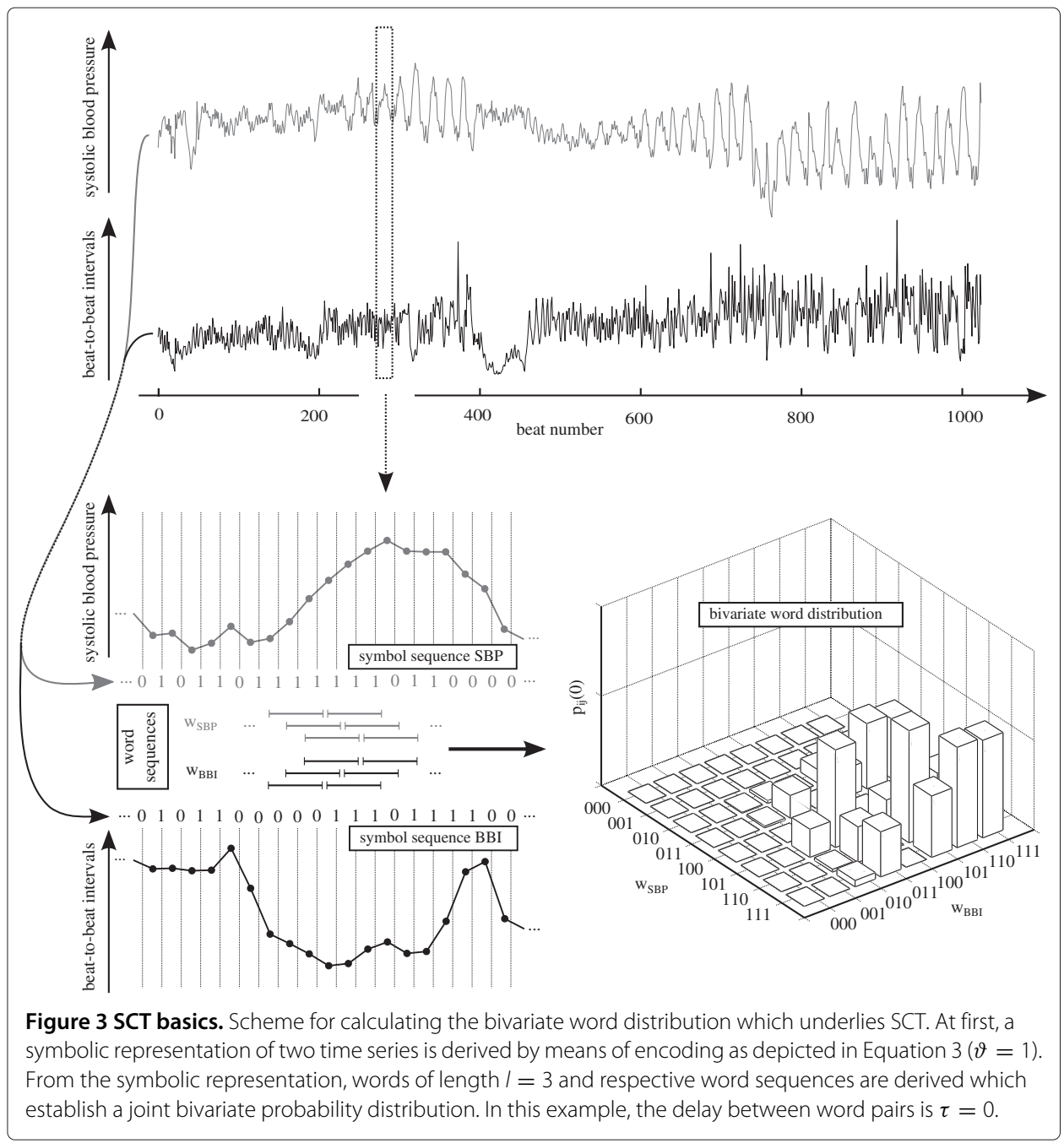

word sequences are considered. The resulting bivariate probability distribution $p_{i j}(\tau)=$ $P\left(w_{x(n)}=W_{i}, w_{y(n+\tau))}=W_{j}\right)$ can be interpreted as a quadratic matrix. By calculating its trace

$$
T=\sum_{i=j} p_{i j}(\tau)
$$

and the diametric fraction

$$
\bar{T}=\sum_{i=1, \ldots, d, j=d+1-i} p_{i j}(\tau)
$$

where $d$ is the number of different patterns; one can derive the coupling parameter $\Delta T$ by

$$
\Delta T=T-\bar{T}
$$

$\Delta T$ is a measure of the strength of a coupling ( $\Delta T>0$ describes symmetric couplings, $\Delta T<0$ describes diametric couplings). The significance of found couplings is assessed by incorporating surrogate data. Details regarding this process and SCT in general can be found in [21] and [14], respectively. Significant couplings for $\tau<0$ can be attributed to the HR driving the BP whereas the BP is assumed to drive the HR for couplings with $\tau \geq 0$. 
For the current work, a displacement of $\theta=1$ to construct the symbol sequences, a word length $l=3$, and lags $\tau=-5, \ldots, 5$ were used.

\section{Test regime and statistics}

We carried out transversal (cross-sectional) and longitudinal analyses. The transversal analysis focused on differences between surgical interventions at identical time instants, i.e., groups MVR, AVR, and TAVI were compared at preOP, $1 \mathrm{~d}$ postOP, and $7 \mathrm{~d}$ postOP. The longitudinal analysis aimed at the temporal development (from preOP over $1 \mathrm{~d}$ postOP to $7 \mathrm{~d}$ postOP) within each surgical intervention.

In both settings, we used Kruskal-Wallis tests to check for significant group differences. Wilcoxon-Mann-Whitney $U$ tests were used for pairwise post hoc tests where KruskalWallis tests revealed significant differences. In the case of the longitudinal setting, we avoided to use a repeated measures procedure as the overlapping data otherwise would have forced us to exclude all patients of whom at least one of the three measurements was not usable/available (which could be attributed to technical, (patho-)physiological, and administrative reasons). An increased probability of a type II error thus should be kept in mind when reading the results [22].

$p$ values less than $0.05,0.01$, and 0.001 were considered as significant, highly significant, and very highly significant, respectively. For pairwise post hoc testing, the significance level was adjusted according to Bonferroni's law to account for multiple comparisons arising from the time instants (preOP, $1 \mathrm{~d}$ postOP, and $7 \mathrm{~d}$ postOP) or from the type of intervention (AVR, MVR, and TAVI), respectively.

\section{Results and discussion}

\section{Results from HRV analysis}

Table 3 summarizes the results from HRV analysis. Figure 4 illustrates selected results. For parameters which showed significant group differences in the Kruskal-Wallis test, the results of transversal post hoc tests are shown above the respective boxes. The results of longitudinal post hoc tests are omitted in the graphic for clarity. These results can be found in Table 4.

Apart from the power ratio $P_{\mathrm{LF}} / P_{\mathrm{HF}}$, there were no differences between the groups in the pre-operative values. The longitudinal analysis proves an apparent decline in autonomic variability for MVR (very highly significant in all parameters apart from the frequency domain parameters). Regarding AVR patients, the decline is not that distinct as in MVR patients but still highly significant for most of the considered HRV parameters. In contrast, TAVI patients do not show any significant difference over the time in none of the evaluated parameters.

Post hoc analysis for prevailing group differences revealed significant differences between preOP and $1 \mathrm{~d}$ postOP and between preOP and $7 \mathrm{~d}$ postOP in most cases. Between $1 \mathrm{~d}$ postOP and $7 \mathrm{~d}$ postOP, no differences were found.

\section{Results from BRS analysis}

Table 5 and Figure 5 show the results concerning the BRS. Analogous to the results regarding the HRV, Figure 5 shows only the results of transversal post hoc tests. The results of longitudinal post hoc tests can be found in Table 4 . 
Table 3 Results from HRV analysis

\begin{tabular}{|c|c|c|c|c|c|c|c|c|}
\hline \multirow{2}{*}{ Parameter } & \multirow{2}{*}{ Group } & \multicolumn{2}{|c|}{ preOP } & \multicolumn{2}{|c|}{$1 \mathrm{~d}$ postOP } & \multicolumn{2}{|c|}{$7 d$ postOP } & \multirow{2}{*}{$p$ value } \\
\hline & & mean $\pm s d$ & $p$ value & mean \pm sd & $p$ value & mean $\pm s d$ & $p$ value & \\
\hline meanNN & MVR & $879 \pm 155$ & & $728 \pm 78.9$ & & $747 \pm 78.7$ & & $<0.001$ \\
\hline \multirow[t]{2}{*}{ (in ms) } & AVR & $902 \pm 138$ & n.s. & $739 \pm 117$ & n.s. & $790 \pm 145$ & n.s. & $<0.001$ \\
\hline & TAVI & $883 \pm 139$ & & $827 \pm 161$ & & $817 \pm 122$ & & n.s. \\
\hline sdNN & MVR & $36.5 \pm 23.8$ & & $14.3 \pm 7.07$ & & $15.1 \pm 9.80$ & & $<0.001$ \\
\hline \multirow[t]{2}{*}{ (in ms) } & AVR & $31.9 \pm 13.6$ & n.s. & $23.1 \pm 10.2$ & $<0.01$ & $25.9 \pm 21.5$ & $<0.001$ & $<0.01$ \\
\hline & TAVI & $28.3 \pm 10.9$ & & $32.5 \pm 13.7$ & & $28.4 \pm 13.5$ & & n.s. \\
\hline shannon & MVR & $1.83 \pm 0.52$ & & $1.03 \pm 0.35$ & & $1.06 \pm 0.42$ & & $<0.001$ \\
\hline \multirow[t]{2}{*}{ (unitless) } & AVR & $1.78 \pm 0.38$ & n.s. & $1.41 \pm 0.43$ & $<0.001$ & $1.42 \pm 0.55$ & $<0.001$ & $<0.001$ \\
\hline & TAVI & $1.63 \pm 0.36$ & & $1.77 \pm 0.34$ & & $1.65 \pm 0.38$ & & n.s. \\
\hline FORBWORD & MVR & $31.7 \pm 13.0$ & & $49.0 \pm 6.07$ & & $47.5 \pm 10.6$ & & $<0.001$ \\
\hline \multirow[t]{2}{*}{ (unitless) } & AVR & $34.4 \pm 10.9$ & n.s. & $36.7 \pm 13.7$ & $<0.001$ & $41.9 \pm 11.1$ & $<0.01$ & $<0.01$ \\
\hline & TAVI & $36.8 \pm 9.72$ & & $27.4 \pm 13.3$ & & $33.1 \pm 17.4$ & & n.s. \\
\hline POLVAR10 & MVR & $0.11 \pm 0.24$ & & $0.43 \pm 0.33$ & & $0.43 \pm 0.27$ & & $<0.001$ \\
\hline \multirow[t]{2}{*}{ (unitless) } & AVR & $0.12 \pm 0.19$ & n.s. & $0.24 \pm 0.27$ & $<0.001$ & $0.24 \pm 0.32$ & $<0.001$ & $<0.05$ \\
\hline & TAVI & $0.07 \pm 0.10$ & & $0.04 \pm 0.05$ & & $0.08 \pm 0.12$ & & n.s. \\
\hline$P_{\mathrm{HF} / P}$ & MVR & $0.14 \pm 0.08$ & & $0.17 \pm 0.14$ & & $0.14 \pm 0.11$ & & n.s. \\
\hline \multirow[t]{2}{*}{ (unitless) } & AVR & $0.13 \pm 0.09$ & n.s. & $0.20 \pm 0.17$ & n.s. & $0.16 \pm 0.15$ & n.s. & n.s. \\
\hline & TAVI & $0.20 \pm 0.12$ & & $0.23 \pm 0.16$ & & $0.23 \pm 0.17$ & & n.s. \\
\hline$P_{\mathrm{LF} / P}$ & MVR & $0.29 \pm 0.12$ & & $0.16 \pm 0.10$ & & $0.13 \pm 0.09$ & & $<0.001$ \\
\hline \multirow[t]{2}{*}{ (in ms) } & AVR & $0.25 \pm 0.10$ & n.s. & $0.20 \pm 0.10$ & n.s. & $0.19 \pm 0.10$ & n.s. & $<0.05$ \\
\hline & TAVI & $0.25 \pm 0.13$ & & $0.16 \pm 0.08$ & & $0.22 \pm 0.14$ & & n.s. \\
\hline$P_{\mathrm{LF}} / P_{\mathrm{HF}}$ & MVR & $2.83 \pm 1.97$ & & $1.70 \pm 2.26$ & & $2.14 \pm 3.28$ & & $<0.01$ \\
\hline \multirow[t]{2}{*}{ (unitless) } & AVR & $3.00 \pm 2.19$ & $<0.05$ & $1.97 \pm 2.54$ & n.s. & $2.90 \pm 2.58$ & n.s. & $<0.05$ \\
\hline & TAVI & $1.93 \pm 1.72$ & & $1.00 \pm 0.62$ & & $1.60 \pm 1.30$ & & n.s. \\
\hline
\end{tabular}

Mean values and standard deviations of HRV parameters. Given $p$ values are results from Kruskal-Wallis tests. The first three $p$ values target transversal comparisons, and the last $p$ value (last column) shows the results of the longitudinal, i.e., within surgical procedure, analysis. Results of transversal post hoc tests for selected parameters are shown in Figure 4. Results of longitudinal post hoc tests are given in Table 4.

The characteristics of BRS are very similar to the ones obtained from HRV analysis: MVR is associated with a strong depression. A less pronounced depression is found for AVR, and TAVI shows no depression at all.

\section{Results of analysis by means of SCT}

Figure 6 shows the results concerning SCT. Both MVR and AVR patients show a typical behavior previous to the surgical intervention consisting of two significant couplings: the symmetric lag at $\tau=0$ is considered to reflect the mechanically and neurally induced fluctuations from respiration. The diametric lag at $\tau=-2$ represents a vagal feedback from the BBI to the SBP [14]. TAVI patients, however, show only one significant coupling in the pre-operative analysis, namely a diametric lag at $\tau=-2$. Considering the longitudinal analysis, the couplings in MVR and AVR patients are completely suppressed at $1 \mathrm{~d}$ postOP. A partial recovery can be observed 7 days after surgery. At $7 \mathrm{~d}$ postOP, the lags $\tau=0$ and $\tau=-2$ have recovered in the case of MVR and AVR, respectively. In TAVI patients, however, there evolve specific couplings until three significant couplings at lags $\tau=1, \tau=-1$, and $\tau=-2$ can be found at $7 \mathrm{~d}$ postOP.

\section{Discussion}

Longitudinal analysis proves an apparently depressed autonomic function after surgery for MVR and AVR. The depression is expressed by decreased meanNN, sdNN, shannon, 


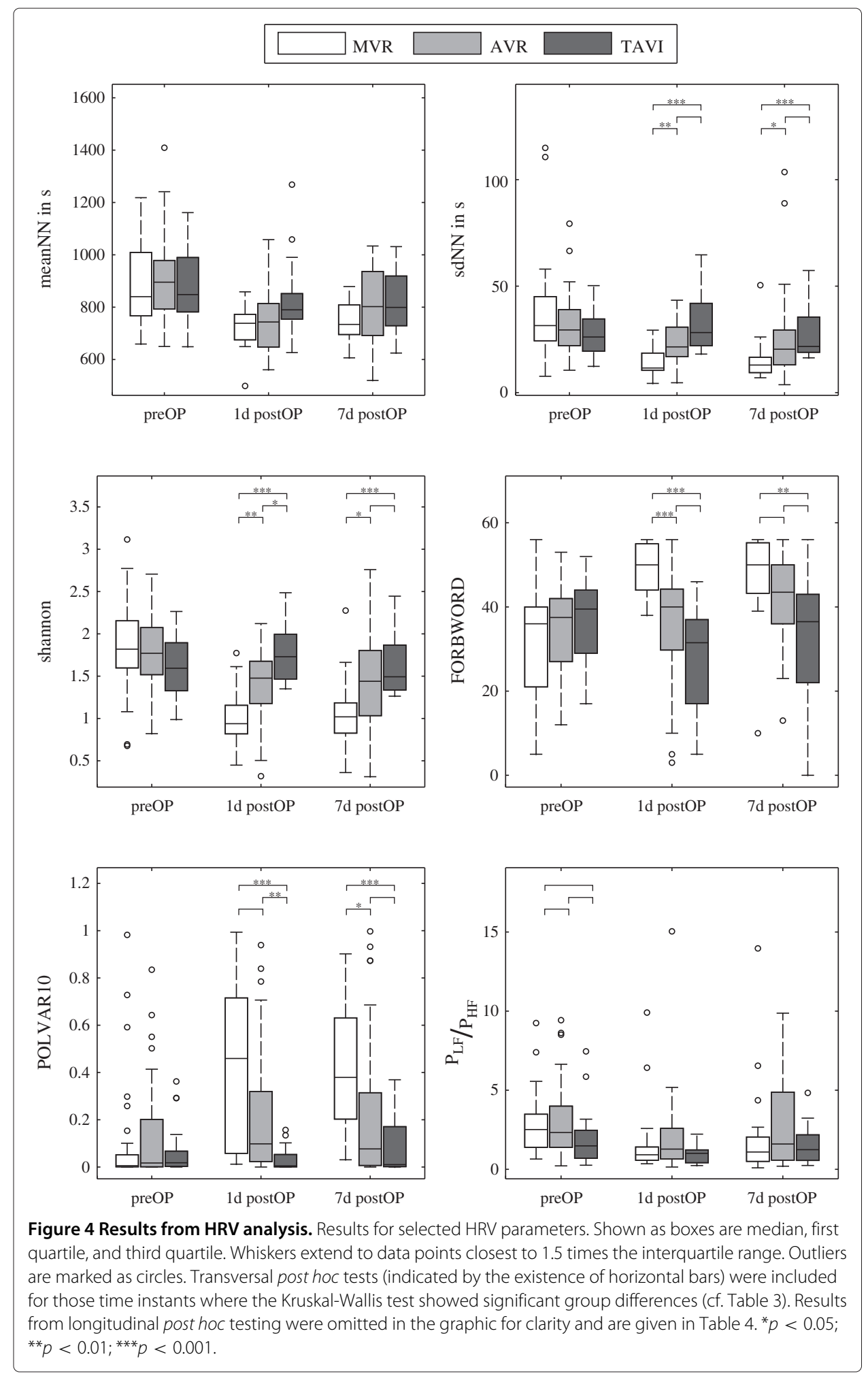

$P_{\mathrm{LF}} / P, P_{\mathrm{LF}} / P_{\mathrm{HF}}, \mathrm{BRS}_{\mathrm{tachy}}$, and $\mathrm{BRS}_{\mathrm{brady}}$ and increased values of symbolic parameters FORBWORD and POLVAR10 (in MVR patients, $p<0.001$ for all time domain HRV and BRS parameters). A decreased cardiovascular variability is consistent to the findings described 
Table 4 Results of longitudinal testing

\begin{tabular}{|c|c|c|c|c|c|c|c|c|c|}
\hline \multirow{2}{*}{ Parameter } & \multicolumn{3}{|c|}{ MVR } & \multicolumn{3}{|c|}{ AVR } & \multicolumn{3}{|c|}{ TAVI } \\
\hline & $\begin{array}{l}\text { preOP vs } \\
1 \mathrm{~d} \text { postOP }\end{array}$ & $\begin{array}{l}\text { preOP vs } \\
7 d \text { postOP }\end{array}$ & $\begin{array}{l}\text { 1d postOP vs } \\
\text { 7d postOP }\end{array}$ & $\begin{array}{l}\text { preOP vs } \\
1 \mathrm{~d} \text { postOP }\end{array}$ & $\begin{array}{l}\text { preOP vs } \\
7 d \text { postOP }\end{array}$ & $\begin{array}{l}\text { 1d postOP vs } \\
\text { 7d postOP }\end{array}$ & $\begin{array}{l}\text { preOP vs } \\
1 \mathrm{~d} \text { postOP }\end{array}$ & $\begin{array}{l}\text { preOP vs } \\
7 d \text { postOP }\end{array}$ & $\begin{array}{l}1 \mathrm{~d} \text { postOP vs } \\
7 \mathrm{~d} \text { postOP }\end{array}$ \\
\hline meanNN & **** & ** & n.s. & **** & ** & n.s. & & No group difference & \\
\hline sdNN & $* * *$ & $* * *$ & n.s. & $* *$ & $* *$ & n.s. & & No group difference & \\
\hline shannon & $* * *$ & $* * *$ & n.s. & $* * *$ & $* *$ & n.s. & & No group difference & \\
\hline FORBWORD & $* * *$ & $* * *$ & n.s. & n.s. & $*$ & n.s. & & No group difference & \\
\hline POLVAR10 & *** & *** & n.s. & * & n.s. & n.s. & & No group difference & \\
\hline$P_{\mathrm{HF}} / P$ & & No group differ & & & No group differ & & & No group difference & \\
\hline$P_{\mathrm{LE} / P}$ & *** & $* * *$ & n.s. & n.s. & * & n.s. & & No group difference & \\
\hline$P_{\mathrm{LF}} / P_{\mathrm{HF}}$ & $* *$ & $*$ & n.s. & $* *$ & n.s. & n.s. & & No group difference & \\
\hline BRS brady & $* * *$ & $* * *$ & n.s. & $* *$ & $* * *$ & n.s. & & No group difference & \\
\hline BRS tachy $_{\text {tact }}$ & *** & $* * *$ & n.s. & n.s. & *** & $*$ & & No group difference & \\
\hline
\end{tabular}

Results of longitudinal post hoc testing for HRV and BRS parameters. The results complement Figures 4 and 5 , respectively. ${ }^{*} p<0.05 ;{ }^{* *} p<0.01 ;{ }^{* * *} p<0.001$. 
Table 5 Results from BRS analysis

\begin{tabular}{|c|c|c|c|c|c|c|c|c|}
\hline \multirow{2}{*}{ Parameter } & \multirow{2}{*}{ Group } & \multicolumn{2}{|c|}{ preOP } & \multicolumn{2}{|c|}{ 1d postOP } & \multicolumn{2}{|c|}{ 7d postOP } & \multirow{2}{*}{$p$ value } \\
\hline & & mean $\pm s d$ & $p$ value & mean $\pm \mathrm{sd}$ & $p$ value & mean \pm sd & $p$ value & \\
\hline BRS brady & MVR & $8.96 \pm 3.25$ & & $4.89 \pm 2.12$ & & $4.92 \pm 3.40$ & & $<0.001$ \\
\hline \multirow[t]{2}{*}{ (in $\mathrm{ms} / \mathrm{mmHg}$ ) } & AVR & $8.44 \pm 3.02$ & n.s. & $6.06 \pm 4.41$ & $<0.05$ & $5.70 \pm 3.06$ & $<0.01$ & $<0.001$ \\
\hline & TAVI & $9.80 \pm 4.63$ & & $7.65 \pm 3.22$ & & $8.67 \pm 4.41$ & & n.s. \\
\hline $\mathrm{BRS}_{\text {tachy }}$ & MVR & $10.0 \pm 3.88$ & & $5.60 \pm 2.91$ & & $4.46 \pm 2.74$ & & $<0.001$ \\
\hline \multirow[t]{2}{*}{ (in $\mathrm{ms} / \mathrm{mmHg}$ ) } & AVR & $8.99 \pm 3.44$ & n.s. & $7.84 \pm 3.79$ & $<0.01$ & $5.92 \pm 2.65$ & $<0.001$ & $<0.001$ \\
\hline & TAVI & $9.20 \pm 3.51$ & & $9.71 \pm 3.83$ & & $9.55 \pm 4.04$ & & n.s. \\
\hline
\end{tabular}

Mean values and standard deviations of BRS parameters. Given $p$ values are results from Kruskal-Wallis tests. The first three $p$ values target transversal comparisons, and the last $p$ value (last column) shows the results of the longitudinal, i.e., within surgical procedure, analysis. Results of transversal post hoc tests are shown in Figure 5). Results of longitudinal post hoc tests are given in Table 4.

by Brown and coworkers who reported a depressed HRV and BRS right after coronary artery bypass graft surgery $[23,24]$. The depression of autonomic function manifests in all parameters related to HRV and BRS apart from $P_{\mathrm{HF}} / P$. For $P_{\mathrm{HF}} / P$, no significant behavior was found. A possible interpretation could be an overall decline of the spectral power by which $P_{\mathrm{HF}}$ is normalized to obtain $P_{\mathrm{HF}} / P$. A decrease in $P$ can mask a specific highfrequency behavior. However, $P_{\text {LFnu }}$ which is also normalized by the spectral power does show a decrease. This finding hints at a stronger decrease of $P_{\mathrm{LF}}$ compared to the decrease in $P_{\mathrm{HF}}$ which is supported by the decreasing ratio $P_{\mathrm{LF}} / P_{\mathrm{HF}}$. Further evidence for a stronger impairment of the sympathetic than that of the parasympathetic branch is given by the

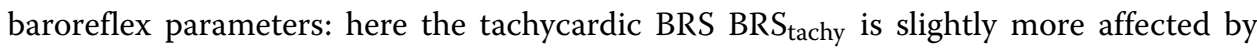
surgery than $\mathrm{BRS}_{\text {brady }}$ which is vagally mediated. A potential explanation in terms of electrophysiology and innervation of the heart could be the vagal innervation being predominantly present in the endocardium, while sympathetic fibers end at both the epiand endocardium [25]. By affecting predominantly the surface of the heart, a stronger influence on the sympathetic system could be the consequence.

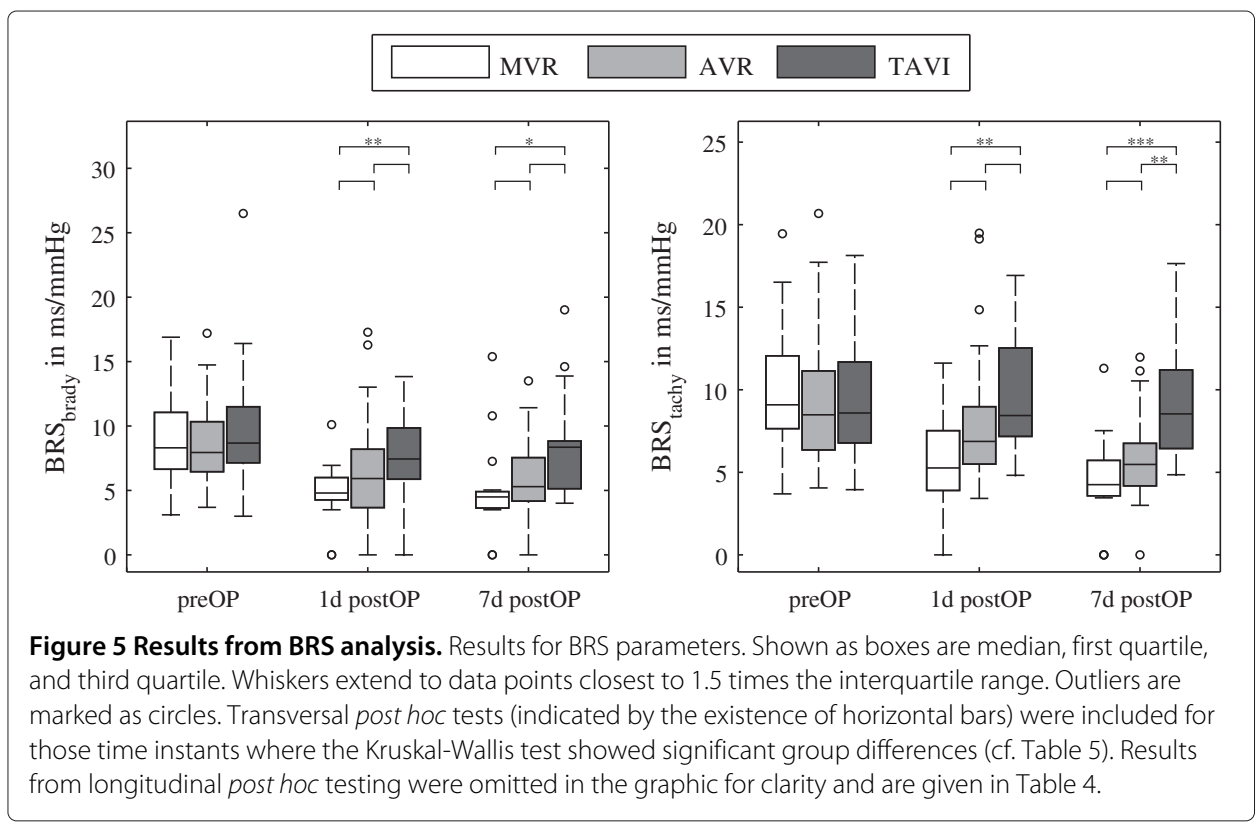




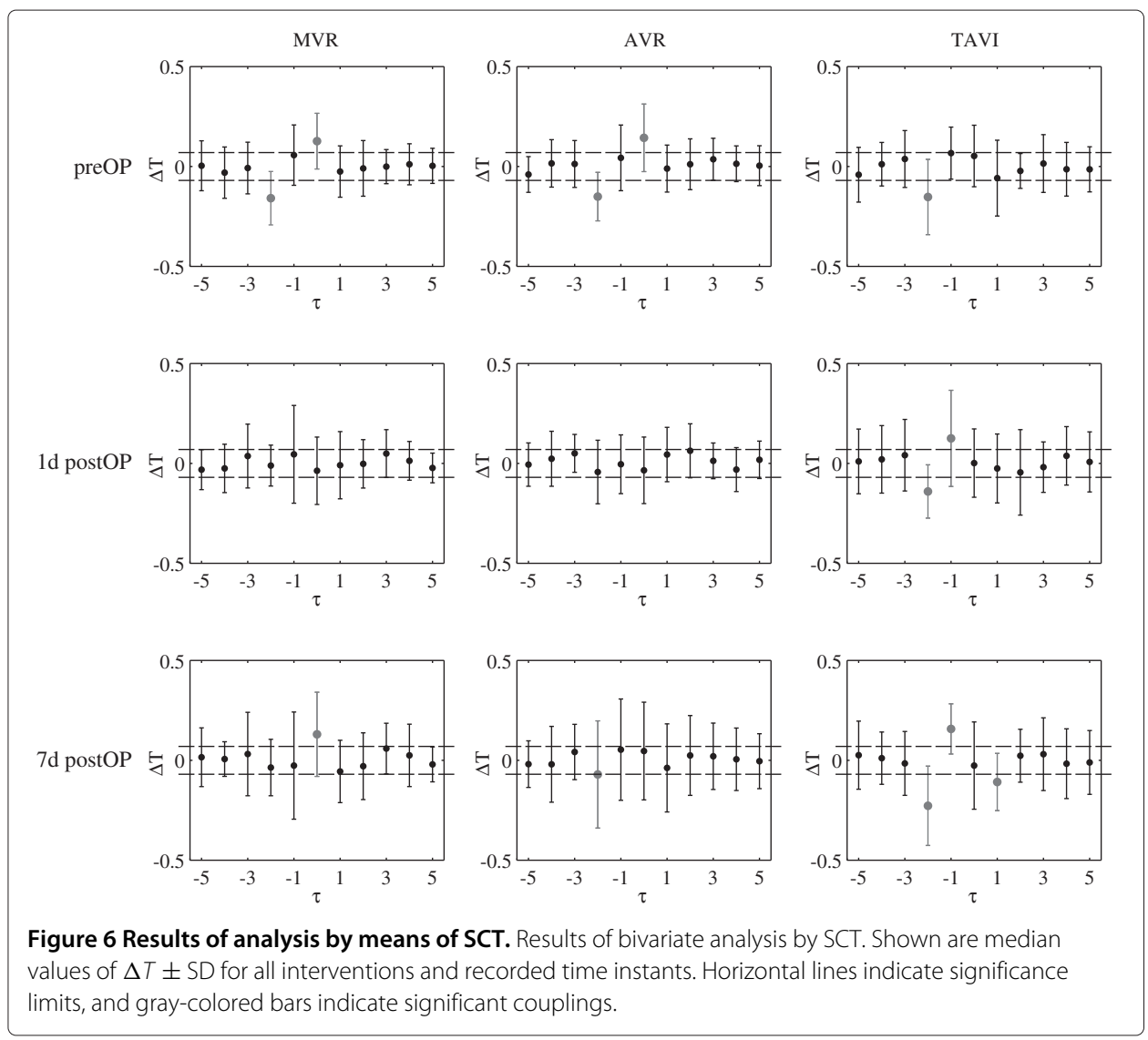

A recovery effect as described by Brown et al. [24], i.e., in our setup a recreation of autonomic function between $1 \mathrm{~d}$ postOP and $7 \mathrm{~d}$ postOP, was not consistently observed, neither MVR patients nor AVR patients. Statistically, this is expressed by non-significant post hoc tests between $1 \mathrm{~d}$ postOP and $7 \mathrm{~d}$ postOP within the longitudinal analysis. Most likely, this is due to the monitored time frame of only 7 days. The reported recovery of autonomic function was related to a 12-week observation interval [24]. The results published by Soares et al. [6] support this hypothesis by describing a drop in autonomic function right after coronary artery surgery which is most distinct 6 days after surgery. A recovery is observed 30 days after surgery. Johansson et al. [7] report an ongoing impaired baroreflex function 5 weeks after coronary artery bypass grafting. A partial recovery is found after a 5-month interval. In spite of differing time intervals, there is much evidence for a long-term recovery to occur. A non-existing recovery at $7 \mathrm{~d}$ postOP, thus, must not be misinterpreted as a sign of missing recovery at all. In fact, some recovery is expected and a long-term follow-up of our patients would have been of high interest in order to relate the short-term findings to the long-term development and, maybe, throw light on mechanisms which affect the duration and degree of the long-term recovery. However, our investigation focused on the short-term impact, and a long-term follow-up was not considered by the study design.

TAVI patients, in contrast, show a behavior which differs from MVR and AVR patients: TAVI does not introduce significant longitudinal changes at all. This indicates the benefit of using TAVI in terms of maintaining the cardiovascular autonomic function compared 
to AVR. The more favorable behavior can be attributed to different factors - TAVI does not require the use of the heart-lung machine nor a cardioplegic arrest of the heart. Moreover, the time of anesthesia is significantly shortened compared to AVR as indicated by Table 1 . Notably, the TAVI group showed no significant decline in HRV and BRS, although the mean age was significantly higher than in patients undergoing MVR and AVR, respectively (the difference arises from the current consensus on using TAVI in high-risk patients). Due to the effects of aging, the TAVI group could be assumed to be prone to cardiovascular instability and impairment by the surgical intervention. However, this is not the case which in our opinion strengthens the assumption of TAVI being a cardiovascular-compliant intervention. These results can be seen in line with the impairment after coronary artery bypass which previously was shown to vary with the degree of invasiveness of the intervention [26].

As regards transversal comparisons, the quantitative behavior of MVR and AVR turns out to be of high interest. Though both interventions cause a depression of cardiovascular autonomic function, the effect is much more pronounced for MVR. The time of anesthesia and operational factors, as factors contributing to the differences between TAVI and MVR as well as TAVI and AVR, respectively, cannot readily explain the differing behavior. In fact, MVR must be assumed as the graver of both interventions. In MVR the caval veins are extensively dissected and the heart is opened by an incision right posterior to the interatrial groove, where an abundance of autonomic nerve endings are supposed to be. For AVR the heart is not strongly affected but the valve is approached by an incision in the anterior aspect of the ascending aorta only. Our results clearly reflect this difference in terms of a more pronounced impairment in the case of MVR. As clinical factors did not differ significantly (see Table 1) and as the handling of patients was standardized between groups of MVR and AVR, these results strongly suggest direct surgical trauma to be responsible for the decrease in HRV and BRS.

SCT provides additional insights compared to the parameters describing HRV and BRS. For MVR and AVR, the result of SCT are in line with the behavior which can found in HRV and BRS parameters: starting from typical couplings at $\tau=0$ and $\tau=-2[14,21,27]$, we found a heavy impairment of autonomic function which manifests in a loss of significant couplings for MVR and AVR 1 day after surgery. Such a reduction of coupling indicates a reduced regulatory capability, thus confirming the findings of HRV and BRS. The partial recovery at $7 \mathrm{~d}$ postOP is difficult to interpret, but it indicates different effects of MVR and AVR on the mechanisms underlying the two typical coupling terms.

For TAVI again a different behavior was observed. After a depressed coupling prior to surgery, significant couplings evolve over time. The missing coupling at lag $\tau=0$ might be related to the higher age and a lower ejection fraction of the group. Both factors can be assumed to alter the respiratory-induced blood pressure variation. A preoperatively reduced normalized low-frequency power of blood pressure variability as compared to AVR patients is in line with this observation [13].

A distinct explanation for the post-operative development is missing and its interpretation owing to the small sample size and again the short observation interval hardly possible. We assume that the general mechanisms which take effect during respiratory sinus arrhythmia are recovered by implanting the aortic valve. As a consequence, an increased blood supply during inspiration causes an acceleration of the heart rate. Factors related to the age and again a limited ejection fraction might cause the observed 
phase delay (not the typical lag at $\tau=0$ is found) which can indicate that respiratoryinduced blood pressure variations are existent but affect the peripheral measurement site by one beat displaced. However, evolving couplings generally suggest a recovery of autonomic functions after a successful surgical intervention. Further studies should confirm our hypotheses by an extended analysis of the recovery period.

\section{Conclusions}

It was shown that cardiac surgery is not only related to a decline of cardiovascular autonomic function but that there is a strong relation between the type of intervention and its effects on the cardiovascular variability and coupling, respectively. In particular, TAVI proved to be beneficial in terms of maintaining the autonomic function compared to AVR. Moreover, a more pronounced suppression of cardiovascular variability in the case of MVR compared to AVR identified direct surgical trauma as a key factor for the resulting impairment of the autonomic function. Further studies incorporating larger populations should confirm our findings and relate the autonomic state to malignant events after surgical interventions to build the fundament of a strengthened inclusion of cardiovascular variability and coupling analysis in the pre-, peri-, and post-operative care.

Competing interests

The authors declare that they have no competing interests.

\section{Authors' contributions}

SZ drafted the manuscript, carried out the statistical analysis, and worked on the interpretation of current results. MR prepared the patient information and the parameters of HRV, BRS, and SCT and worked on the interpretation of current results. JK, HM, and RB co-developed the study design and provided the methodical basis for the analysis which we carried out. NW co-developed the study design, co-drafted the preceding papers where the analysis of autonomic function was conducted in a pairwise setting, and worked on the interpretation of current results. All authors read and approved the final manuscript.

\section{Acknowledgements}

This study was supported by grants from the Deutsche Forschungsgemeinschaft (BA 1581/4-1, BR 1303/8-1, KU 837/20-1, BA 1581/6-2, BR 1303/8-3, KU-837/29-2) as well as the European projects EU NEST-pathfinder and BRACCIA.

\section{Author details}

${ }^{1}$ Institute of Biomedical Engineering, TU Dresden, Helmholtzstraße 18, Dresden 01069, Germany. ${ }^{2}$ Department of Physics, Humboldt-Universität zu Berlin, Berlin 10115, Germany. ${ }^{3}$ Transdisciplinary Concepts and Methods, Potsdam Institute for Climate Impact Research, Potsdam 14473, Germany. ${ }^{4}$ Department of Thoracic and Cardiovascular Surgery, University of Ulm, Ulm 89081, Germany.

Received: 6 August 2013 Accepted: 3 February 2014

Published: 1 April 2014

\section{References}

1. La Rovere MT, Bigger JT, Marcus Fl, Mortara A, Schwartz PJ: Baroreflex sensitivity and heart-rate variability in prediction of total cardiac mortality after myocardial infarction. ATRAMI (Autonomic Tone and Reflexes After Myocardial Infarction) Investigators. Lancet 1998, 351(9101):478-484.

2. La Rovere MT, Pinna GD, Hohnloser SH, Marcus FI, Mortara A, Nohara R, Bigger JT, Camm AJ, Schwartz PJ: Baroreflex sensitivity and heart rate variability in the identification of patients at risk for life-threatening arrhythmias implications for clinical trials. Circulation 2001, 103(16):2072-2077.

3. Cole CR, Blackstone EH, Pashkow FJ, Snader CE, Lauer MS: Heart-rate recovery immediately after exercise as a predictor of mortality. New England J Med 1999, 341(18):1351-1357. doi:10.1056/NEJM199910283411804.

4. Laitio TT, Huikuri HV, Kentala ES, Mäkikallio TH, Jalonen JR, Helenius H, Sariola-Heinonen K, Yli-Mäyry S, Scheinin H: Correlation properties and complexity of perioperative RR-interval dynamics in coronary artery bypass surgery patients. Anesthesiology 2000, 93(1):69-80.

5. Bauernschmitt R, Malberg H, Wessel N, Kopp B, Schirmbeck EU, Lange R: Impairment of cardiovascular autonomic control in patients early after cardiac surgery. Eur J Cardiothorac Surg 2004, 25(3):320-326. doi:10.1016/j.ejcts.2003.12.019.

6. Soares PPS, Moreno AM, Cravo SLD, Nóbrega ACL: Coronary artery bypass surgery and longitudinal evaluation of the autonomic cardiovascular function. Crit Care (London, England) 2005, 9(2):124-131. doi:10.1186/cc3042.

7. Johansson M, Karlsson A-K, Myredal A, Lidell E: Arterial baroreflex dysfunction after coronary artery bypass grafting. Interact Cardiovasc Thorac Surg 2009, 8(4):426-430. doi:10.1510/icvts.2008.198747.

8. Demirel $S, A k k a y a ~ V$, Oflaz H, Tükek T, Erk O: Heart rate variability after coronary artery bypass graft surgery: a prospective 3-year follow-up study. Ann Noninvasive Electrocardio/ 2002, 7(3):247-250. 
9. Lakusic N, Slivnjak V, Baborski F, Sonicki Z: Heart rate variability in patients after cardiac valve surgery. Cen Eur J Med 2008, 3(1):65-70. doi:10.2478/s11536-007-0070-y.

10. Walther T, Falk V, Kempfert J, Borger MA, FassI J, Chu MWA, Schuler G, Mohr FW: Transapical minimally invasive aortic valve implantation; the initial 50 patients. Eur J Cardiothorac Surg 2008, 33(6):983-988. doi:10.1016/j.ejcts.2008.01.046.

11. Webb JG, Chandavimol M, Thompson CR, Ricci DR, Carere RG, Munt BI, Buller CE, Pasupati S, Lichtenstein S: Percutaneous aortic valve implantation retrograde from the femoral artery. Circulation 2006, 113(6):842-850. doi:10.1161/CIRCULATIONAHA.105.582882.

12. Retzlaff B, Bauernschmitt R, Malberg H, Brockmann G, Uhl C, Lange R, Kurths J, Bretthauer G, Wessel N: Depression of cardiovascular autonomic function is more pronounced after mitral valve surgery: evidence for direct trauma. Philos Transact A Math Phys Eng Sci 2009, 367(1892):1251-1263. doi:10.1098/rsta.2008.0272.

13. Retzlaff B, Wessel N, Riedl M, Gapelyuk A, Malberg H, Bauernschmitt N, Kurths J, Bretthauer G, Bauernschmitt R: Preserved autonomic regulation in patients undergoing transcatheter aortic valve implantation (TAVI): a prospective, comparative study. Biomed Tech (Berl) 2011, 56(4):185-193. doi:10.1515/BMT.2011.017.

14. Wessel N, Suhrbier A, Riedl M, Marwan N, Malberg H, Bretthauer G, Penzel T, Kurths J: Detection of time-delayed interactions in biosignals using symbolic coupling traces. EPL (Europhysics Lett) 2009, 87(1):10004.

15. Bleiziffer S, Ruge H, Mazzitelli D, Schreiber C, Hutter A, Laborde J-C, Bauernschmitt R, Lange R: Results of percutaneous and transapical transcatheter aortic valve implantation performed by a surgical team. Eur J Cardiothorac Surg 2009, 35(4):615-620. discussion 620-621. doi:10.1016/j.ejcts.2008.12.041.

16. Wessel N, Malberg H, Bauernschmitt R, Kurths J: Nonlinear methods of cardiovascular physics and their clinical applicability. I J Bifurcation Chaos 2007, 17(10):3325-3371.

17. Malik M: Heart rate variability. Standards of measurement, physiological interpretation, and clinical use. Task Force of the European Society of Cardiology and the North American Society of Pacing and Electrophysiology. Eur Heart J 1996, 17(3):354-381.

18. Voss A, Kurths J, Kleiner HJ, Witt A, Wessel N: Improved analysis of heart rate variability by methods of nonlinear dynamics. J Electrocardio/ 1995, 28 Suppl:81-88.

19. Bertinieri G, di Rienzo M, Cavallazzi A, Ferrari AU, Pedotti A, Mancia G: A new approach to analysis of the arterial baroreflex. J Hypertens Supp/ 1985, 3(3):79-81.

20. Malberg H, Wessel N, Hasart A, Osterziel K-J, Voss A: Advanced analysis of spontaneous baroreflex sensitivity, blood pressure and heart rate variability in patients with dilated cardiomyopathy. Clin Sci (Lond) 2002, 102(4):465-473.

21. Suhrbier A, Riedl M, Malberg H, Penzel T, Bretthauer G, Kurths J, Wessel N: Cardiovascular regulation during sleep quantified by symbolic coupling traces. Chaos 2010, 20(4):45124. doi:10.1063/1.3518688.

22. Bedeian AG, Feild $\mathrm{HS}$ : Assessing group change under conditions of anonymity and overlapping samples. Nursing Res 2002, 51(1):63-65.

23. Brown CA, Wolfe LA, Hains S, Ropchan G, Parlow J: Spontaneous baroreflex sensitivity after coronary artery bypass graft surgery as a function of gender and age. Can J Physiol Pharmacol 2003, 81(9):894-902. doi:10.1139/y03-087.

24. Brown $C A$, Wolfe $L A$, Hains $S$, Ropchan G, Parlow J: Heart rate variability following coronary artery bypass graft surgery as a function of recovery time, posture, and exercise. Can J Physiol Pharmacol 2004, 82(7):457-464. doi:10.1139/y04-076

25. Marron K, Wharton J, Sheppard MN, Fagan D, Royston D, Kuhn DM, de Leval MR, Whitehead BF, Anderson RH, Polak $J M$ : Distribution, morphology, and neurochemistry of endocardial and epicardial nerve terminal arborizations in the human heart. Circulation 1995, 92(8):2343-2351.

26. Hogue CW, Stein PK, Apostolidou I, Lappas DG, Kleiger RE: Alterations in temporal patterns of heart rate variability after coronary artery bypass graft surgery. Anesthesiology 1994, 81(6):1356-1364.

27. Penzel T, Riedl M, Gapelyuk A, Suhrbier A, Bretthauer G, Malberg H, Schöbel C, Fietze I, Heitmann J, Kurths J, Wesse $\mathrm{N}$ : Effect of CPAP therapy on daytime cardiovascular regulations in patients with obstructive sleep apnea. Comput Biol Med 2012, 42(3):328-334. doi:10.1016/j.compbiomed.2011.09.001.

Cite this article as: Zaunseder et al:: Impact of cardiac surgery on the autonomic cardiovascular function. Journal of Computational Surgery 2014 1:9. 